\title{
Les enjeux de la mondialisation
}

The hazards of globalization

Los retos de la globalización

\section{Cecilia Braslavsky}

Traducteur : Rozenn Dedeyan

\section{(2) OpenEdition}

12 Journals

Édition électronique

URL : http://journals.openedition.org/ries/2373

DOI : $10.4000 /$ ries. 2373

ISSN : 2261-4265

Éditeur

Centre international d'études pédagogiques

Édition imprimée

Date de publication : 1 octobre 2000

Pagination : 33-49

ISSN : 1254-4590

\section{Référence électronique}

Cecilia Braslavsky, « Les enjeux de la mondialisation », Revue internationale d'éducation de Sèvres [En ligne], 27 | 2000, mis en ligne le 01 octobre 2003, consulté le 10 décembre 2020. URL : http:// journals.openedition.org/ries/2373; DOI : https://doi.org/10.4000/ries.2373

\section{(c) Tous droits réservés}




\title{
Les enjeux de la mondialisation 1
}

\author{
Cecilia Braslavsky ${ }^{2}$
}

\section{Résumé}

Les processus de globalisation en cours depuis quelques années devraient bouleverser les structures des systèmes éducatifs. L'aggravation des inégalités sociales, la permanence des changements accélérés et incontrôlés générés par la primauté absolue de l'économique déstabilisent brutalement les systèmes éducatifs et sont en contradiction avec les valeurs humanistes que ceux-ci affichent généralement. Les tensions augmentent quotidiennement se manifestant souvent par des situations de violence scolaire.

\section{The hazards of globalization}

The process of globalization that has been in progress for some years is likely to have a drastic effect on the structure of education systems. The deepening of social inequalities and the rapid, uncontrolled and lasting changes produced by the absolute predominance of the economy are savagely destabilizing education systems and go against the humanistic values that these latter generally support. Tension is increasing daily as often witnessed in acts of violence in schools.

\section{Los retos de la globalización}

Los procesos de globalización que ocurren desde algunos años deberían trastornar las estructuras de los sistemas educativos. La recrudescencia de las desigualdades sociales, la permanencia de cambios acelerados y no controlados, resultado de la primacía absoluta de lo económico, están destabilizando los sistemas educativos y contradicen sus valores generalmente humanistas. Las tensiones que aumentan cada día se manifiestan a menudo de manera violenta en el ámbito escolar.

1 Cet article a été traduit par Rozenn Dedeyan.

2 Les opinions exprimées dans cet article sont celles de l'auteur et n'engagent aucune institution. 
En 1989, le monde est entré dans le xxı e siècle ${ }^{3}$. À partir de cette date, les processus en cours sous toutes les latitudes se sont articulés et interpénétrés encore plus étroitement que par le passé. Les processus de globalisation, entamés dès les débuts des temps modernes, au Xve siècle, se sont approfondis et étendus à un point encore inimaginable il y a peu. Ce mouvement est si puissant que nombreux sont ceux qui se demandent si les systèmes éducatifs nationaux continueront d'exister.

En effet, les systèmes éducatifs tels que nous les connaissons se sont développés sur les bases de l'utopie et des processus de développement de sociétés modernes à structure pyramidale et qui avaient vocation à l'intégration. Actuellement, on assiste à l'émergence de sociétés qui tendent à se structurer " en sablier", dont les parties supérieure et inférieure se séparent de plus en plus et tendent à fonctionner en vase clos : l'une avec tous les avantages et les paradoxes du développement post-industriel; l'autre avec tous les problèmes d'exclusion de ce développement et avec très peu de possibilités de trouver des solutions.

Dans ce contexte de passage de sociétés pyramidales exclusives à une société en sablier et à une perspective de rouages à plusieurs vitesses, il n'y a pas de demandes abstraites à l'éducation. Il y a différentes configurations de demandes en fonction des positions politiques et idéologiques à partir desquelles s'interprète ce type de société et de perspective.

Une de ces perspectives nie la viabilité d'une humanité qui évolue à des vitesses multiples, mais au sein de laquelle les mouvements de chacun de ces rouages peuvent déstabiliser l'autre. En d'autres termes, selon cette perspective, il apparaît que dans un monde de plus en plus interconnecté, ceux qui participent des rouages de préjudices ne supporteront pas les différences avec ceux qui participent des rouages de bénéfices et, inversement, ceux qui participent des rouages de bénéfices n'accepteront pas de les distribuer. Dans cette perspective, les deux groupes s'annihileront à travers des épisodes de violence successifs. L'humanité sera en conflit permanent et pourrait même s'effondrer.

Le moyen d'éviter cela est de lutter pour construire une société à un seul cercle, qui remplacerait la société en pyramide. Il s'agit de parvenir à ce que tous participent d'un même tissu social, et non de différents tissus parallèles; dans ce schéma, les personnes devraient se placer, à certains moments de leur vie, dans des zones plus centrales de ce cercle et, à d'autres, dans des zones plus périphériques, mais sans êtres exclues et en maintenant la possibilité de circulation.

Le propos du présent article est de tenter de réfléchir sur la forme que prennent les tendances de développement contemporaines, et sur les défis qu'elles représentent pour les systèmes éducatifs et les institutions déjà établies

3 E. Hobsbawm, Historia del siglo XX, Espagne, Ed. Crítica, 1995. 
sur les bases de "l'utopie du cercle et de la circulation ». Il n'y a aucune prétention à l'originalité, mais plutôt une tentative de réactualisation du débat. Cet article s'organise en trois parties. La première expose les grandes tendances mentionnées et les défis posés pour l'éducation. La seconde tente de systématiser certaines des réponses les plus efficaces, et la troisième présente des exemples de conflits et de tensions provoqués par les changements. Pour finir, on proposera une réflexion sur certains débats larvés qui existent, sans exister encore tout à fait, dans le dialogue éducatif.

Les analyses et les réflexions concernent davantage l'enseignement secondaire que l'enseignement primaire, ceci à cause de la place centrale qu'occupe le premier dans le travail de réflexion et dans les débats éducatifs de la fin du xxe siècle, et sans doute du début du Xxie siècle, mais aussi parce que dans le monde entier, il est bien plus difficile d'innover à ce niveau en voie de massification qu'au niveau de l'enseignement primaire, ou même universitaire.

\section{Développement contemporain et éducation}

Ce chapitre aborde les principales tendances du développement contemporain qui aujourd'hui touchent tous les continents et tous les peuples ${ }^{4}$, en essayant dans chaque cas d'en tirer les conséquences pour l'éducation.

\section{Un monde du travail hétérogène, décroissant et mouvant}

À l'heure actuelle, pas moins de cinq processus économiques se produisent dans le monde entier. Le premier concerne la diminution du volume de travail disponible et même nécessaire pour la satisfaction des besoins essentiels des sociétés. Il est donc possible que les jeunes, actuellement en formation, attendent de plus en plus avant d'entrer sur le marché du travail et passent par des périodes de chômage ou de sous-emploi. Le second confirme une augmentation des emplois disponibles dans le secteur des services par rapport au secteur agricole et industriel. Le troisième touche l'augmentation du travail informel. Le quatrième consiste en un changement de plus en plus rapide des profils de métiers, notamment en termes de compétences spécifiques. Le cinquième concerne la modification des échelles sur lesquelles se réalise et se résout la situation professionnelle des personnes.

Les concepts de globalisation et d'internationalisation du marché du travail font référence aux manifestations actuelles de changements entamés depuis longtemps, mais qui se sont récemment fortement accélérés. La globali-

4 R. Ortiz, Otro Territorio. Ensayos sobre el mundio contemporáneo. Santa Fé de Bogotá, Convenio Andrés Bello, 1998. 
sation et l'internationalisation du travail signifient, entre autres choses, que le capital aussi bien que les entreprises et les travailleurs ont la possibilité de passer d'un pays à l'autre afin de mieux répondre aux offres et aux demandes respectives.

Ces processus économiques représentent différents défis pour l'éducation. La diminution du volume du travail disponible pose de façon urgente le problème du retard de l'entrée sur le marché du travail et de l'allongement de la scolarité obligatoire, englobant toutes les strates de l'offre éducative destinées aux jeunes et aux adolescents. Par ailleurs, la rapidité des changements de profils professionnels remet de plus en plus à l'ordre du jour la vieille demande pédagogique d' "apprendre à apprendre ", et en fait un impératif socio-économique et personnel, alors même que la perte de la capacité de création d'emploi des économies agricole et industrielle amène à repenser la formation professionnelle et à réclamer qu'elle soit étroitement associée à la formation aux services et aux emplois de bureau, y compris au sein de la production de biens agricoles et industriels. Enfin, la croissance du secteur informel par rapport au secteur formel de l'économie donne à penser qu'il serait extrêmement souhaitable que tous les jeunes apprennent à entreprendre.

Dans l'ensemble, les nouvelles tendances de l'économie obligent à se poser très sérieusement des questions sur le niveau des systèmes éducatifs et les spécialisations précoces. Mais; au-delà de ces questions, les évolutions de l'économie amènent à affirmer aussi la nécessité de repenser la manière dont l'éducation, et particulièrement l'éducation secondaire, s'articule à l'économie. Est-il nécessaire, possible et même souhaitable que l'éducation accepte de former des jeunes pour un marché du travail qui n'a apparemment pas de place pour tous?

Au cours de la dernière décennie, on a assisté au renforcement de la position selon laquelle l'éducation formelle ne doit pas assumer la responsabilité de la formation au monde du travail, tel qu'il est organisé à un moment donné, mais la formation au travail. Il est probable qu'il faille même poser le principe que l'éducation - dans son ensemble - doit former à la multiactivité ${ }^{5}$ : au travail productif, à la création culturelle, à une vie sociale harmonieuse, à la vie familiale, etc. ; et - contrairement à ce qui s'est passé tout au long du xxe siècle - à l'alternance entre des périodes dominées par tel ou tel type d'activité au cours d'une même vie. S'il en est ainsi, il faudrait néanmoins se préoccuper en même temps de résoudre la question de la formation à des travaux spécifiques qui nécessitent des compétences tout aussi spécifiques, alors même que ces métiers risquent de disparaître ou de se transformer très rapidement.

5 A. Gorz, Miserias del presente, riqueza de lo posible, Buenos Aires, Paidós, 1998. 


\section{L'aggravation des inégalités sociales}

Par ailleurs, une des caractéristiques les plus complexes, aux conséquences moins prévisibles, des nouvelles modalités du développement économique est l'aggravation des inégalités sociales existantes et l'émergence de nouvelles inégalités. Selon divers auteurs, dans les sociétés contemporaines, le point de départ de chaque individu serait de plus en plus déterminant pour le reste de sa vie. La mobilité sociale serait très faible et, chez les enfants et les jeunes, les différences de possibilité d'accumulation de capital éducatif seraient telles qu'elles détermineraient des inégalités de situations finales encore plus grandes qu'elles ne l'étaient au point de départ ${ }^{6}$. Dans ces conditions, il serait très difficile de réaliser l'harmonie sociale, la croissance économique et la démocratie. La violence quotidienne augmenterait en permanence, revêtant des formes comparables à celles d'une espèce de guérilla permanente, mais sans finalité politique de réforme ou de révolution sociale ${ }^{7}$.

S'il est difficile de croire que l'on peut remédier à ce genre de situation par des décisions d'ordre exclusivement éducatif, il semble particulièrement important, dans ce contexte, de concevoir des structures et des processus éducatifs qui garantissent au moins des chances de formation équivalentes, prennent en compte la diversité des points de départs et luttent en permanence contre la prétendue détermination irréversible des destinées éducatives des individus, sans ignorer les limites d'une telle politique.

La structure en niveaux des systèmes éducatifs des XIXe et $\mathrm{XX}^{\mathrm{e}}$ siècles supposait que les élites n'avaient besoin d'aucune formation liée aux activités de production et de distribution de biens et de services et que - de leur côté - les travailleurs manuels, y compris moyennement ou hautement qualifiés, n'avaient aucunement besoin d'approfondir leurs connaissances en matière de fonctionnement socio-économique, ni d'étendre leurs horizons culturels. Mais les récents progrès scientifiques et technologiques sont en train, peu à peu, de transformer les métiers de telle sorte que les professions manuelles tendent à disparaître en tant que choix de vie au profit de solutions robotisées dans de vastes secteurs de l'économie ; tandis que dans d'autres, des économies submergées de type très ancien se maintiendront. Ceux qui resteront dans ce type d'économies n'auront guère de chances d'améliorer leur qualité de vie.

6 J.P. Fitoussi, P. Rosanvallon, La nueva era de las desigualdades, Buenos Aires, Ediciones Manantial, 1997 ; 0. Altimir, "Desigualdad, empleo y pobreza en América Latina : efectos del ajuste y del cambio en el estilo del desarrollo ", in : Desarrollo Económico, n 145, vol. 37, Instituto de Desarrollo Económico y Social, Buenos Aires, 1997 ; A. Minujín, " ¿La gran exclusion? Vulnerabilidad y exclusión en América Latina ", in : D. Filmus, (comp.), Los noventa. Política, sociedad y cultura en América Latina y Argentina de fin de siglo, Buenos Aires, Flacso/Eudeba, 1999.

7 J. Rifkin, El fin del trabajo, Buenos Aires, Paidós, 1996. 
En effet, tout laisse augurer que de plus en plus, le travail associé à des possibilités de meilleure qualité de vie nécessitera une formation intellectuelle solide, mais rénovée, et une orientation tout aussi solide et rénovée vers la résolution de problèmes, c'est-à-dire l'action. Dans ce contexte, il nous faut relever un triple défi : arriver à une meilleure répartition du peu de «bon » travail existant, promouvoir la mise en place de passerelles entre celui-ci et d'autres activités moins liées au progrès technique et, enfin, faciliter la transformation d'activités appartenant au secteur submergé de l'économie en activités qui intègrent le progrès technique et permettent l'amélioration de la qualité de vie. Pour relever ces trois défis de façon systémique et avec le moins d'effets contraires possibles, il faut que chaque individu développe les compétences nécessaires pour se tirer d'affaire dans le contexte du progrès économique, sans en négliger les conséquences. De plus, ces trois défis présentent une particularité - nous le répétons, au risque de lasser - celle de se jouer au niveau international. La facilité technologique pour le développement de migrations massives, la construction d'une idéologie de la liberté de mouvement nécessaire pour l'économie et son impact sur la légitimation de la mobilité des personnes exigent que ceci soit rapidement compris, et que l'on agisse en conséquence, par des politiques éducatives de plus en plus internationales.

La combinaison d'une solide formation générale comprenant des humanités, des sciences et de la technologie, avec des méthodologies pédagogiques de contextualisation ${ }^{8}$ serait la seule solution pour que tous les adolescents et les jeunes puissent avoir accès aux types d'activités susceptibles de leur assurer une meilleure qualité de vie et pour lesquels ils devront à la fois savoir penser et savoir faire. Pour cela il faut que tous les parcours éducatifs possibles comprennent des humanités et des enseignements technologiques d'une part, et des possibilités de formation concrète à la résolution de problèmes, d'autre part. En outre, cette combinaison permettrait plus facilement à tous d'apprendre à penser mieux et à faire mieux. En effet, il y aurait dans le monde des évidences empiriques croissantes de ce que la combinaison des deux types d'apprentissages améliore la qualité de chacun d'eux et permet de chercher de nouvelles solutions à des problèmes qui n'en ont pas encore.

La conception de propositions pédagogiques basées sur des expériences de formation à forte composante situationnelle et de résolution de problèmes, a des racines historiques dans les processus de construction des disciplines scolaires, mais c'est une alternative qui a rencontré des résistances tendancielles. Selon certains auteurs ${ }^{9}$, les efforts en vue de bâtir une éducation - et surtout

8 Araujo, Oliveira, La nueva empresa : el aprendizaje como parte del negocio, Montevideo, CINTERFOR, 1994 ; C. De Moura Castro, Educación vocacional y productividad : alguna luz en la caja negra, Centro nacional de recursos humanos, Brasilia, IPEA,1984.

9 I. Goodson, Historia del currículum. La construcción social de las disciplinas escolares, Barcelone, Ediciones Pomared-Corredor, 1995. 
une éducation secondaire - plus contextualisée n'ont pu aboutir au XIXe siècle, parce que la victoire de cette conception aurait signifié la dévalorisation de l'accès et de la permanence dans l'éducation secondaire comme instrument de différenciation entre les élites et les travailleurs. La nouvelle prise de conscience de la valeur des connaissances entraînera de nouveaux mouvements dans la dynamique de contrôle de leur distribution. En conséquence, le passage au type d'éducation proposé avive certains conflits d'intérêts et en entraine de nouveaux. La tension est si vive qu'en de nombreuses occasions, les réformes, particulièrement au niveau secondaire, s'avèrent «impossibles » ${ }^{10}$.

Le caractère conflictuel des réformes exige de ceux qui mènent ces changements une forte capacité d'argumentation, de concertation et de conception de formes appropriées de contrôle, en fonction des conditions particulières à chaque pays et à l'échelle régionale et mondiale.

Cet aspect du problème est lié à une autre dimension de la formation à laquelle il est nécessaire de donner la priorité. Il s'agit de renforcer la formation aux valeurs de telle sorte que les jeunes comprennent et acceptent la nécessité de cohésion et rejettent la polarisation sociale. En d'autres termes, il faut faire en sorte que l'éducation promeuve la construction d'une forte conscience collective du fait qu'une distribution inégale et arbitraire des possibilités d'accès aux biens sociaux, en particulier à l'éducation et au travail, ne constitue pas seulement une atteinte aux droits fondamentaux de quelques-uns, mais un risque pour la survie de tous.

\section{Un contexte de changement accéléré}

Historiquement, les systèmes éducatifs ont été pensés pour "transmettre la culture des générations adultes aux jeunes générations ", et ceci plus encore dans le cas de l'éducation secondaire que dans le cas de l'éducation primaire. Cette position reposait sur cinq hypothèses qui sont actuellement discutables. La première est que les jeunes générations ne possèdent pas de culture propre et, en conséquence, n'opposent aucune résistance à l'apprentissage de contenus et reprennent automatiquement à leur compte les modèles culturels des adultes; la seconde est que la culture adulte est homogène ; la troisième est que les jeunes générations sont elles aussi homogènes; la quatrième est que la culture adulte et, en tant qu'élément de cette culture, les caractéristiques de la production et des structures de connaissances sont stables dans le temps; la cinquième est que les systèmes éducatifs et les écoles sont les principaux systèmes experts dans le domaine de la transmission de l'information.

Au cours des dernières années, on a assisté à la reconnaissance de l'hétérogénéité culturelle et à la reconnaissance des cultures historiquement 
assujetties, en particulier les cultures d'origine ${ }^{11}$. En conséquence de cette reconnaissance, on voit apparaître dans de nombreux pays une demande de prise en compte de cette diversité, qui s'exprime par exemple à travers la possibilité d'inclure l'enseignement des langues originaires dans les programmes. Là où cette reconnaissance est refusée, de graves conflits apparaissent, qui conduisent à des guerres et à des génocides, la rendant encore plus nécessaire.

On observe également dans de nombreux pays du monde un processus croissant d'émergence et de renforcement de cultures de jeunes, à travers des productions et des consommations qui passent par des circuits différents des circuits scolaires ${ }^{12}$. Les résultats de certaines recherches rendent compte du fait que l'inadaptation du modèle de l'école secondaire aux caractéristiques des nouveaux groupes et secteurs sociaux qui la fréquentent, ainsi que le manque de prise en considération de l'existence de besoins et de demandes propres aux jeunes expliquent en grande partie l'importance du taux d'absentéisme et d'échec scolaire ${ }^{13}$.

Par conséquent, pour que les enfants et les jeunes viennent, restent et apprennent dans les institutions éducatives, il faut qu'ils y trouvent des possibilités d'épanouissement grâce à des pratiques variées qui permettent de faire des écoles des espaces de vie raisonnable. Mais, en plus, ces enfants et ces jeunes ne sont pas un conglomérat homogène, mais un ensemble de groupes de personnes avec des intérêts, des besoins et des savoirs divers, qui convergent ou divergent les uns par rapport aux autres et par rapport aux adultes.

D'autre part, l'éducation secondaire est apparue à une époque où les savoirs se structuraient en disciplines académiques distinctes les unes des autres, et dans une perspective de permanence dans le temps. Avec l'émergence d'un nouveau système scientifico-technologico-productif ${ }^{14}$, on assiste également à l'effacement et la redéfinition permanents des frontières entre les disciplines académiques et la mise en place d'importantes articulations internes qui, cependant, sont d'une durée limitée. Dans ce contexte, de grands champs disciplinaires structurés pendant la deuxième moitié du xxe siècle sont restés en-dehors de l'éducation secondaire tandis que des disciplines d'une actualité douteuse en font encore partie.

Enfin, la révolution des communications a ouvert la voie à de nouveaux systèmes experts dans la transmission et l'accès à l'information qui remplissent ces fonctions beaucoup plus efficacement que les systèmes éducatifs et les

11 F. Calderón, M. Dos Santos, Sociedades sin atajos, cultura, politica y reestructuración económica en América Latina, Buenos Aires, Paidós, 1995 ; Jesús Martin-Barbero, De los medios a las mediaciones. Comunicación, Cultura y Hegemonía, Santa Fé de Bogotá, Convenio Andrés Bello, 1998 (5e édition).

12 P. Semán, P. Vila, « Rock chabón e identidad juvenil en la Argentina neo-liberal », in : D. Filmus (comp.), op. cit. 13 G. A. Obiols, S. di Segni de Obiols, Adolescencia, posmodernidad y escuela secundaria. La crisis de la enseñanza media, Buenos Aires, Kapelusz, 1994.

14 J. Lesourne, Educación y Sociedad. Los desafíos del año 2000, Barcelone, Gedisa, 1993. 
collèges ${ }^{15}$. En conséquence, il est indispensable que les institutions éducatives prennent conscience des risques et des possibilités que représente pour elles l'existence de ces nouveaux systèmes experts, en particulier à travers l'émergence du concept de connectivité des intelligences ${ }^{16}$.

L'idée selon laquelle les politiques éducatives se sont chargées de réinventer des processus d'institutionnalisation dans des conditions de désaffiliation institutionnelle ${ }^{17}$ qui comportent des risques graves pour l'épanouissement de la personne, la cohésion sociale et la paix, est particulièrement séduisante - bien que source de conflits.

\section{La recherche d'approfondissement démocratique}

Par ailleurs, au cours des dernières décennies, un grand nombre de pays ont entamé des processus de démocratisation. Mais dans le même temps, de larges secteurs sociaux qui vivent sous des régimes démocratiques sont en quête de nouvelles solutions pour transformer les pratiques politiques, c'est-àdire toutes les définitions et les actions liées à la chose publique. Cette demande est associée à la crise de la représentation et de la légitimité des modes particuliers d'exercice de la politique, aux changements qui interviennent dans les formes d'insertion économique, à l'augmentation de l'exclusion sociale, aux restructurations du monde des communications. De cet ensemble de données, a émergé le modèle de la "société de marché ». Selon certains, comme tout est matière à transaction, seules se développent vigoureusement des stratégies individualistes de succès, réfractaires à des engagements collectifs et sources de malaise et de désillusion ${ }^{18}$. Pour d'autres, en revanche, surgissent alors d'autres formes de participation, liées à la consommation comme forme d'exercice du pouvoir ${ }^{19}$.

Dans tous les cas, les hypothèses impliquent la nécessité d'une nouvelle prise de position forte en ce qui concerne la démocratie et sa relation avec l'éducation. Il y a quelques décennies, dans la majeure partie du monde, lorsqu'on cherchait les articulations entre démocratie et éducation, la première était asso-

15 R. Pallof, K. Pratt, Building Learning Communities in Cyberspace. Effective Strategies for the Online Classroom, San Francisco, Jossey-Bass, 1999 ; L. Harasim, S. Hiltz, L. Teles, M. Turoff, Learning Networks, Cambridge, The Mit Press, 1998.

16 D. De Kerckhove, Connected Intelligence. The Arrival of the Web Society, Toronto, Somerville House Publishing, 1997.

17 M. Castells, "Las metamorfosis de la cuestión social ", Buenos Aires, Ed. Paidós, 1997.

18 N. Lechner, "Las condiciones de la gobernalidad democrática en América Latina de fin de siglo », in :

D. Filmus (comp.), op. cit.

19 A. Minc, www.capitalisme.fr, Paris, Grasset, 2000. 
ciée exclusivement aux formes institutionnelles du libéralisme démocratique. Dans ce contexte, les défis éducatifs consistaient essentiellement à former les électeurs, les représentants et les fonctionnaires d'un état de droit.

Actuellement les possibilités de surmonter le malaise et la désillusion sont associées à l'élargissement du concept de démocratie ou à sa transformation en un concept différent, susceptible de réactiver la capacité à reconstruire des engagements collectifs à partir de pratiques sociales, identifiées historiquement comme telles, et de pratiques économiques qui prennent de plus en plus d'importance sur le plan social.

Dans ce cadre, les perspectives de participation de l'éducation à la démocratie augmentent. La défense et la promotion des droits de l'homme et de nouveaux droits sociaux, économiques et de la personne sont au cour du concept même de démocratie.

À partir de cet élargissement du concept, de nouveaux thèmes sont de plus en plus à l'ordre du jour en tant que défis pour l'éducation. Parmi ces thèmes, citons l'enseignement des droits de l'homme d'un point de vue conceptuel et concret, le respect des différences et l'intégration des jeunes ayant des besoins éducatifs spéciaux.

Les jeunes eux-mêmes semblent s'inscrire dans une tension entre une désillusion apparente vis-à-vis de la politique et un dynamisme qui s'exprime dans leur façon d'intervenir dans la vie associative et publique, les mouvements sociaux, culturels et religieux qui attirent un plus grand nombre et une plus grande variété d'institutions et de personnes, et les blanchissent de l'accusation d'apathie lancée par ceux qui ne conçoivent d'autres formes légitimes de participation à la vie politique que celles, visibles, qu'ils connaissent dans le cadre du système des partis.

La possibilité que ces nouvelles formes d'action trouvent de nouvelles voies d'institutionnalisation et s'instaurent durablement dans des espaces adéquats pour la promotion solidaire et partagée d'une meilleure qualité de vie dépend aussi, pour une large mesure, de ce que les personnes possèdent des capacités et des valeurs appropriées et pour cela, qu'elles disposent de plus de temps et de nouvelles offres de formation.

\section{Vers l'utopie de l'intégration}

Face à ces nouvelles tendances évoquées plus haut, on peut identifier un ensemble de propositions et de lignes directrices des réformes en cours dans différents systèmes éducatifs nationaux, qui sont animées par la volonté politique de contribuer à "l'utopie du cercle» social d'intégration à laquelle nous avons fait référence au début de ce texte.

Ces tendances de changement se rapportent à tous les aspects des systèmes éducatifs, et notamment la structure, les curricula et l'organisation. 
Du point de vue de la structure, les trois tendances les plus importantes sont:

- le remplacement des systèmes éducatifs organisés en niveaux, avec des passages difficiles et exclusifs, par des systèmes éducatifs organisés en cercle, avec des passages en douceur et inclusifs;

- le remplacement des modalités d'éducation secondaire organisées en fonction des secteurs classiques des économies de la première moitié du xxe siècle, par le prolongement d'une éducation de base qui inclue des options organisées pour rendre compte de la diversité des intérêts des jeunes ;

- le remplacement de la professionnalisation précoce associée à certaines de ces modalités par des solutions de formation professionnelle et technique modulaire, menées parallèlement ou à la suite de la dernière étape de la formation de base (qui continue de s'appeler secondaire supérieure et occupe les $10^{\mathrm{e}}, 11^{\mathrm{e}}$ et $12^{\mathrm{e}}$ années de scolarisation obligatoire).

L'éducation primaire et l'éducation secondaire sont à l'origine des voies alternatives d'éducation formelle pour les couches inférieures ou dirigeantes des sociétés européennes. Leurs inventeurs ne les avaient pas conçues comme deux moments à l'intérieur d'un système éducatif structuré en niveaux, mais comme deux segments d'un système éducatif structuré en voies parallèles, pour une société divisée en classes, qu'on pourrait représenter de façon graphique comme des blocs superposés et quasiment sans interconnections ni possibilités de passer de l'un à l'autre. Cet ensemble de blocs superposés n'avait même pas la forme de la pyramide à laquelle nous faisions allusion plus haut, mais plutôt celle d'un large rectangle inférieur placé horizontalement - correspondant à l'école primaire surmonté d'un mince rectangle intermédiaire vertical, se terminant en pointe. Les lycées français et les Gymnasien allemands, par exemple, formaient le mince rectangle intermédiaire, et l'université la pointe supérieure.

L'émergence de classes moyennes en Europe et la transformation progressive de cette société des "États » de l'Ancien Régime en une pyramide de classes avec des possibilités d'ascension sociale constituent un phénomène qui a commencé vers le milieu du XIXe siècle et s'est accéléré après la seconde guerre mondiale et jusqu'aux années soixante-dix, entraînant alors une longue période de reconfiguration ou de réorganisation des systèmes éducatifs. Cette reconfiguration s'est axée sur le positionnement de l'éducation secondaire dans le prolongement de l'éducation primaire, en superposant deux modèles institutionnels très différents et sans que l'un ou l'autre n'ait été réformé en profondeur ${ }^{20}$.

Au niveau du secondaire, cette reconfiguration s'est orientée vers une spécialisation de l'éducation selon trois modalités correspondant aux trois secteurs des économies industrielles arrivées à maturité, et qui devaient assurer la

20 D. Mueller, F. Ringer, B. Simon, El desarrollo del sistema educativo moderno. Cambio estructural y reproducción social 1870-1920, Madrid, Ministerio del Trabajo y Seguridad, 1992. 
formation des individus destinés à intégrer le secteur intermédiaire de la pyramide : formation commerciale pour les services du même nom, formation industrielle pour le travail manufacturier et les écoles agro-techniques pour les métiers qui utilisaient le progrès technique dans le secteur primaire des économies.

Dès les années cinquante, en Europe une idée faisait son chemin : celle de l'école comprehensive ou polyvalente, de longue durée, destinée non seulement à former tous les citoyens, mais encore à former des citoyens rendus égaux par le biais d'une éducation commune à tous ${ }^{21}$. Cette idée s'était fait jour à partir des premiers diagnostics portés sur la "révolution scientifique et technologique», de l'intuition de changements progressifs dans la structure des métiers et de la volonté de promouvoir la redistribution des chances en matière de travail et d'insertion sociale. Derrière cette idée se profilait l'utopie de l'édification d'une société circulaire, dans laquelle les personnes ne resteraient pas fixées en un point donné dans une structure de classes rigide.

Si l'idée de l'école polyvalente devait au début rencontrer de fortes résistances, elle fut ensuite promue pour faciliter le lancement de passerelles entre différents parcours éducatifs et des processus d'homologation des premières années d'une éducation secondaire longue.

Par ailleurs, au cours des dernières décennies, des changements se sont produits aussi autour de la question de la différenciation horizontale à l'intérieur de l'offre éducative destinée aux jeunes et aux adolescents. Comme nous le disions, vers 1950, pratiquement tous les pays du monde disposaient des structures adéquates pour former les jeunes aux métiers de la pyramide de la société industrielle européenne arrivée à maturité. Le diplôme de fin d'études secondaires avait pour but d'assurer aux jeunes une formation propédeutique à caractère logico-linguistico-mathématique pour qu'ils puissent ensuite avoir accès à l'université. Les écoles commerciales, industrielles et normales avaient pour vocation d'offrir aux jeunes une formation professionnelle plus pratique pour qu'ils accèdent plus tard à un emploi de technicien moyen dans l'agriculture, l'industrie, les services commerciaux ou l'enseignement. En Europe et en Afrique, les modalités étaient identiques sur le papier. Parce que contrairement aux sociétés et aux économies, le papier, lui, résiste à tout.

Les deux changements les plus significatifs intervenus depuis 1950 ont consisté, dans un ensemble de pays notamment dans presque toute l'Amérique latine, à transférer la formation des enseignants des écoles primaires du niveau secondaire au niveau supérieur non universitaire ou universitaire, et à ouvrir la voie pour que les jeunes qui sortaient des écoles techniques et agricoles puissent avoir accès à des études supérieures et universitaires. Parallèlement, d'autres solutions de changement devaient n'avoir que peu d'impact : par exemple,

21 D. Hargreaves, The Challenge for the Comprehensive School. Culture, Curriculum and Community, Londres, Routledge, 1982. 
l'introduction de plans d'alternance inspirés du système dual allemand dans les années correspondant au niveau secondaire inférieur.

Le balancement entre innovations réussies et propositions sans effet, dans les années soixante, soixante-dix, et quatre-vingt a encouragé une dissociation de la formation professionnelle de l'éducation secondaire, tendant à promouvoir le transfert de la première vers le niveau supérieur. L'Australie a été un des pays pionniers dans cette direction.

Le prolongement de cette tendance présente au moins trois risques. Le premier est que l'on recommence à concevoir en Afrique, en Asie ou en Amérique latine, des modèles institutionnels qui n'ont rien à voir avec les économies et les sociétés de ces régions; le second est que - surtout dans les pays où il y a le plus de pauvreté - on assiste à la disparition de l'offre gratuite de formation professionnelle pour des métiers qui nécessitent une qualification déterminée. Le troisième est que les jeunes issus des milieux populaires se voient refuser la possibilité d'apprendre mieux grâce à des méthodologies pédagogiques contextualisées dans l'action.

La conséquence de ces changements réside dans le fait que la diversité des structures - jadis considérée comme une hérésie allant à l'encontre de la justice - commence à être envisagée dans certains pays comme une alternative possible pour parvenir à une organisation plus efficace dans des contextes divers.

Parallèlement on a promu "l'autonomie de l'école», qui a tardé à être élaborée, selon deux orientations différentes. La première est liée à la promotion de la liberté de choix. La seconde est associée à la promotion de la créativité pédagogique. Cette seconde tendance prend progressivement d'autres dénominations: «autogestion", "auto-administration", "autonomie de moyens, mais pas de fins ».

Au cours des premières étapes de la promotion de l'autonomie scolaire, dans de nombreux pays, on n'a pas prêté attention à des questions comme l'évaluation, l'information, les curricula et la formation de nouveaux profils professionnels pour une nouvelle forme d'exercice des politiques et des pratiques éducatives. Derrière ce manque d'attention se cachait, selon nous, une perte du sens des finalités de l'éducation en général et de l'éducation secondaire en particulier. À mesure que grandissait la prise de conscience de l'importance de la compétitivité nationale dans le contexte de la globalisation, du progrès technique comme facteur de compétitivité nationale et de la variable éducation comme élément essentiel pour l'introduction du progrès technique, et que les processus de décentralisation progressaient dans de nombreux pays, on a commencé à accorder une plus grande attention aux dispositifs permettant de contrôler la qualité de l'éducation ainsi que la construction et la diffusion de l'information. En conséquence, dans plusieurs pays où il n'existait pas de systèmes d'épreuves standardisées ni de procédures de publication des résultats; on les a créés. L'idée était qu'un public mieux 
informé serait en mesure de prendre de meilleures décisions individuelles favorables à la situation collective.

Mais vers la fin du xxe siècle, il devint évident que la régulation ne garantissait pas, en soi, une meilleure qualité de l'éducation ; et qu'il fallait aussi promouvoir celle-ci. À cause de cela, le curriculum, en tant que cadre d'orientation pour la promotion éducative, devait se repositionner peu à peu comme axe de débats sur les réformes éducatives. Actuellement les questions de curricula sont, sous une forme ou une autre, au centre de la réflexion, des discussions et des politiques éducatives. Que faut-il enseigner ? Comment organiser les propositions ? Comment concilier les demandes issues de toutes les différentes tendances citées précédemment? Comment progresser dans des pays où existent de telles différences?

À partir de ces préoccupations, plusieurs pays ont opéré des changements à différents niveaux. Quatre d'entre eux sont de nature structurelle :

- orienter toute la formation vers certaines compétences de base et intellectuelles supérieures ;

- tenter de réduire la fragmentation en promouvant des pratiques pédagogiques inter et multidisciplinaires ;

- introduire des options pour les élèves dans des systèmes où il n'y en avait pas jusque-là ;

- compenser l'accroissement des marges d'option par la concertation de contenus communs.

D'autres changements concernent la façon d'aborder chaque ensemble de connaissances. Dans de nombreux pays de tous les continents, on a avancé l'idée d'aborder l'enseignement des langues de façon communicative. En mathématiques, les propositions formalistes ont cédé la place à des contenus liés à la probabilité, la statistique et l'utilisation des mathématiques pour formuler des modèles interprétatifs. Dans le domaine des sciences sociales, ce sont les propositions orientées vers l'enseignement des processus, plutôt que vers la transmission de l'information, qui ont gagné du terrain. On a multiplié les efforts pour initier les enfants à la diversité des points de vue et à la capacité à débattre. On a élaboré le concept d'alphabétisation scientifique. Les points de vue environnementalistes ont progressé. Dans le domaine de l'éducation physique, on a abandonné les approches militaristes. Dans l'éducation artistique, on met plus résolument en avant les idées de production et de créativité.

Mais rien de tout cela ne se fait de façon uniforme dans tous les pays, et encore moins sans effort. Le degré et la forme de ces progrès ne sont pas satisfaisants, les résistances sont multiples, les contradictions innombrables.

\section{Les conflits suscités par le changement}

L'expansion et l'évolution de l'éducation suscitent dans le monde entier de multiples conflits, différents d'un pays à l'autre. On a pu se douter à la 
lecture de ce qui précède de la nature de certains d'entre eux; mais il en existe d'autres. Ces conflits, qui peuvent se classer en trois grands groupes, sont liés :

- aux tensions entre éducation et modèles de développement ;

- aux nouveaux points de vue concernant l'éducation des jeunes et les traditions de l'éducation secondaire;

- à l'organisation de l'appareil institutionnel public et à sa manière de promouvoir les changements en matière d'éducation.

Parmi les conflits décrits dans le premier groupe, les tensions se produisent habituellement :

- entre la nécessité d'organiser des espaces d'insertion et d'apprentissage pour tous et les limites des budgets consacrés à l'éducation, et en particulier à l'éducation des jeunes;

- entre la certitude d'un monde d'activités en évolution qui exige une préparation polyvalente et les besoins immédiats de formation pour remplir des fonctions au sein des économies actuelles, qu'elles soient formelles ou informelles ;

- entre les contenus requis par la globalisation et l'ouverture, comme par exemple l'anglais, et ceux qui font l'objet de revendications identitaires et de droit à la différence des peuples originaires, comme les langues de ces peuples;

- entre la reconnaissance de la nécessité de transformer les écoles secondaires en véritables écoles pour les enfants et les jeunes et la peur qu'engendrent ces mêmes enfants et jeunes dans de nombreuses sociétés.

Les professeurs et les anciens modèles pédagogiques sont au centre des conflits liés aux tensions qui existent entre les nouveaux points de vue sur l'éducation des jeunes et les traditions de l'éducation secondaire, par exemple :

- entre la nécessité de transformer la structure en niveaux et les modalités ainsi que l'organisation en disciplines des collèges du secondaire ;

- entre la conviction et le désir de changer des professeurs et leur crainte de perdre leurs droits du travail acquis dans le cadre de mouvements, à plus ou moins long terme, comme récemment la diminution de leur salaire ;

- entre les propositions de contenus et de méthodologies généralement acceptées à cause de leur richesse pour former des besoins d'apprentissage essentiels et les savoirs dont disposent ces mêmes professeurs, qui trouvent ces propositions séduisantes mais reconnaissent qu' "ils ne savent pas comment s'y prendre »;

- entre les demandes et les investissements croissants en formation de professeurs et l'utilisation de méthodologies pédagogiques répétitives et dépassées.

Enfin, il existe un ensemble de tensions liées à la nécessité de libérer la créativité pédagogique des institutions éducatives, les communautés et le corps enseignant, tout en renforçant la capacité d'orientation et de promotion des États nationaux. En effet, l'analyse des réformes éducatives fiscalistes des années quatre-vingt et du début des années quatre-vingt-dix dans plusieurs pays du monde a montré que si l'on recherche la qualité et l'équité dans l'éducation, il 
n'est pas possible de démonter les vieux appareils bureaucratiques des ministères de l'Éducation sans ériger à leur place de nouvelles instances institutionnelles différentes pour orienter la réforme des systèmes éducatifs et l'innovation institutionnelle et promouvoir la créativité. Dans ce sens, il faut trouver de nouvelles formes institutionnelles de direction publique de l'éducation sans reconstruire les modèles pyramidaux, hiérarchiques et autoritaires qui asphyxient la créativité des institutions éducatives, des communautés et du corps enseignant. Les réformes qui sont menées actuellement utilisent parfois des structures anciennes dynamisées par des hégémonies personnelles très fortes, des programmes d'investissement à court terme, ou des structures institutionnelles provisoires. Il n'est, certes, pas souhaitable de revenir aux appareils bureaucratiques pesants et routiniers du passé, mais on ne peut pas se passer d'institutions stables.

\section{Réflexions finales}

À l'évidence, la question de l'attention aux besoins des enfants, des adolescents et des jeunes en matière d'éducation sera un des thèmes prioritaires du programme des premières décennies du Xxie siècle. Il est aussi évident que ce programme aura trois dimensions à la fois différenciées et articulées. La première concerne la nécessité de réinventer les formes d'éducation traditionnellement offertes à la population. La seconde est liée à la nécessité de transformer les institutions qui existent aujourd'hui, et la troisième à la création de davantage de possibilités institutionnelles pour permettre l'intégration de tous.

L'importance que revêtent ces trois dimensions varie beaucoup selon les pays, mais dans tous les cas, il apparaît qu'indépendamment de cette importance et des différences qui existent entre les questions à traiter pour donner des réponses aux problèmes posés partout à cet égard, certains pays, voire certains continents entiers ne disposent pas des ressources nécessaires pour faire face à leurs besoins en matière d'éducation. Par ailleurs, on est en droit de se demander si, dans le contexte actuel de globalisation, chaque pays et chaque région doit être autosuffisant dans le domaine de l'éducation. Une partie non négligeable de la population de ces pays ou régions, en particulier la plus instruite, peut être amenée plus tard à travailler ailleurs. Qui doit financer son éducation?

Mais il faut avancer au-delà de cette réflexion. Dans le contexte actuel du "tout-économique», il est sûrement tentant de penser que la principale carence est d'ordre financier. En réalité le nœud gordien ne se trouve pas exclusivement dans la recherche de moyens économiques et financiers, mais dans la nécessité de générer au sein des sociétés la capacité d'inventer des solutions et de susciter des accords et des mouvements de transformation permettant de maintenir en vie un système d'éducation publique susceptible d'intégrer tous les enfants, les adolescents et les jeunes dans un circuit institutionnel qui facilite 
leur épanouissement personnel. Pour l'heure, dans la majorité des cas, ils ne peuvent trouver place qu'au sein d'institutions inadaptées, vieillies et déconsidérées, qui ne sont que de mauvaises copies d'un modèle adapté à certaines régions développées du monde au milieu du $\mathrm{Xx}^{\mathrm{e}}$ siècle. Parfois ces institutions ne sont même pas accessibles à tous. Mais les enfants et les jeunes se battent quand même pour y aller, parce que, de toute façon, ils n'ont pas de meilleure solution. Ils sont même prêts à en bénéficier au prix de la violence.

$$
\text { ** }
$$

Comment sortir de cette situation? Dans le contexte de la globalisation déshumanisée, de nouvelles réponses apparaissent, qui ne font pas encore l'objet d'un débat sérieux. Deux en particulier :

- d'abord, pour éviter à de nombreux jeunes de participer à cette dégradation, et pour les former de façon à les intégrer au cercle riche et rapide, la meilleure solution est de leur offrir une éducation par le biais d'un usage intensif des nouveaux moyens de communication avec une autonomie d'interaction entre pairs ;

- en second lieu, pour promouvoir leur identité personnelle et diverse, le mieux est qu'ils soient en outre éduqués par des formateurs quasi individuels. Ces propositions n'ont rien d'original. Elles s'inspirent des deux axes du débat pédagogique de toute l'histoire de l'humanité. Le premier se réfère au caractère privé ou public des processus d'éducation. Il ne s'agit pas dans ce cas de savoir si la gestion est à la charge de l'État, des églises ou de d'autres institutions. Il s'agit de décider si les contenus et les mécanismes de répartition des possibilités d'apprentissage doivent être déterminés par les sociétés ou peuvent rester en marge de celles-ci. Le second axe se réfère au profil des éducateurs. C'est le vieux débat entre le professeur de Locke, destiné à former des gentilshommes ; et le maître de Comenio ou l'éducateur de Pestalozzi, faits - chacun à sa façon - pour former tout le monde. 